\title{
Kinetic Resolution of Planar-Chiral Ferrocenes by Molybdenum-Catalyzed Enantioselective Metathesis
}

\author{
Masamichi Ogasawara,* Susumu Watanabe, Liyan Fan, Kiyohiko Nakajima, and Tamotsu Takahashi* \\ Catalysis Research Center and Graduate School of Pharmaceutical Sciences, Hokkaido University, \\ and SORST, Japan Science and Technology Agency (JST), Kita-ku, Sapporo 001-0021, Japan, \\ and Department of Chemistry, Aichi University of Education, Igaya, Kariya, Aichi, 448-8542, Japan
}

\section{Supporting Information}

\section{Experimental Section.}

General. All anaerobic and/or moisture sensitive manipulations were carried out with standard Schlenk techniques under predried nitrogen or with glovebox techniques under prepurified argon. ${ }^{1} \mathrm{H}$ NMR (at 400 $\mathrm{MHz}$ ) and ${ }^{13} \mathrm{C}$ NMR (at $100 \mathrm{MHz}$ ) chemical shifts are reported in ppm downfield of internal tetramethylsilane. Tetrahydrofuran and benzene were distilled from benzophenone-ketyl under nitrogen prior to use. Dichloromethane was distilled from $\mathrm{CaH}_{2}$ under nitrogen prior to use. $\mathrm{Cp} \cdot \mathrm{Na}^{1}, \mathrm{C}_{5} \mathrm{H}_{4}{ }^{t} \mathrm{Bu}_{2},{ }^{2} \mathrm{C}_{5} \mathrm{H}_{4} \mathrm{Cy}_{2},{ }^{3}$ $\mathrm{C}_{5} \mathrm{H}_{4}\left(\mathrm{SiMe}_{3}\right)_{2},{ }^{4} \mathrm{Fe}(\mathrm{acac})_{2},{ }^{5} \mathrm{Mo}\left(=\mathrm{NC}_{6} \mathrm{H}_{3}-2,6-{ }^{i} \mathrm{Pr}_{2}\right)\left(=\mathrm{CHCMe}_{2} \mathrm{Ph}\right)(\mathrm{OTf})_{2}(\mathrm{dme}),{ }^{6}$ 3,3'- ${ }^{t} \mathrm{Bu}_{2}-\mathrm{H}_{8}$-binaphthol $\mathrm{K}_{2}$ salt, ${ }^{7}$ and $\left(\mathrm{H}_{2} \mathrm{IMes}\right)\left(\mathrm{PCy}_{3}\right) \mathrm{Cl}_{2} \mathrm{Ru}(=\mathrm{CHPh})$ (the 2 nd-generation Grubbs' catalyst ${ }^{8}$ were prepared according to the reported methods. All the other chemicals were obtained from commercial sources.

Allylcyclopentadienes. Monosubstituted allylic cyclopentadienes $\left(\mathrm{C}_{5} \mathrm{H}_{5}\right.$-allyl, $\mathrm{C}_{5} \mathrm{H}_{5}$-crotyl, $\mathrm{C}_{5} \mathrm{H}_{5}$-cinnamyl, $\mathrm{C}_{5} \mathrm{H}_{5}-\mathrm{CH}_{2} \mathrm{CH}=\mathrm{CMe}_{2}, \mathrm{C}_{5} \mathrm{H}_{5}$-methallyl) were prepared from $\mathrm{Cp} \cdot \mathrm{Na}$ and the corresponding allylic chlorides or bromides in THF and purified by vacuum distillation prior to use. ${ }^{9}$ Trisubstituted allylcyclopentadienes $\left(\mathrm{C}_{5} \mathrm{H}_{3}(\text { allyl })^{t} \mathrm{Bu}_{2}, \mathrm{C}_{5} \mathrm{H}_{3}(\right.$ allyl $) \mathrm{Cy}_{2}, \mathrm{C}_{5} \mathrm{H}_{3}($ allyl $\left.)\left(\mathrm{SiMe}_{3}\right)_{2}\right)$ were prepared from $\left(\mathrm{C}_{5} \mathrm{H}_{3}-1,3-\mathrm{R}_{2}\right) \cdot \mathrm{Li}\left(\mathrm{R}={ }^{t} \mathrm{Bu}, \mathrm{Cy}\right.$, or $\mathrm{SiMe}_{3}$ ), which were generated from the corresponding $\mathrm{C}_{5} \mathrm{H}_{4} \mathrm{R}_{2}$ and ${ }^{n} \mathrm{BuLi}$ in THF, and allyl bromide and purified by vacuum distillation prior to use. All these cyclopentadiene derivatives were obtained as mixtures of doublebond regioisomers, and thus characterized by GC analyses only.

Racemic Diallylferrocene Substrates (1). ${ }^{10}$ A typical procedure is given for the synthesis of 1a. To a THF $(8 \mathrm{~mL})$ solution of $\mathrm{Fe}(\mathrm{acac})_{2}(2.54 \mathrm{~g}, 10.0 \mathrm{mmol})$ was added a solution of $\left.\left(\mathrm{C}_{5} \mathrm{H}_{2}-1 \text {-allyl-2,4- }{ }^{t} \mathrm{Bu}\right)_{2}\right) \cdot \mathrm{Li}$, which was prepared from $\mathrm{C}_{5} \mathrm{H}_{3}\left(\right.$ allyl ${ }^{t} \mathrm{Bu}_{2}(2.18 \mathrm{~g}, 10.0 \mathrm{mmol})$ and ${ }^{n} \mathrm{BuLi}(1.60 \mathrm{M}$ hexane solution, $6.3 \mathrm{~mL}, 10.1 \mathrm{mmol})$ in THF $(25 \mathrm{~mL})$, at $-78{ }^{\circ} \mathrm{C}$ and the mixture was stirred at $0{ }^{\circ} \mathrm{C}$ for $1 \mathrm{~h}$. After cooling the mixture to $-78{ }^{\circ} \mathrm{C}$, to this was added a solution of $\left(\mathrm{C}_{5} \mathrm{H}_{4}\right.$-allyl) $\cdot \mathrm{Na}$, which was prepared from $\mathrm{C}_{5} \mathrm{H}_{5}$-allyl $(1.06 \mathrm{~g}, 10.0 \mathrm{mmol})$ and $\mathrm{NaH}$ $(240 \mathrm{mg}, 10.0 \mathrm{mmol})$ in THF $(10 \mathrm{~mL})$. The resulting mixture was stirred at room temperature for $3 \mathrm{~h}$. The mixture was diluted with hexane and filtered through a pad of Celite. After removal of the solvent, the remaining dark-red oil was purified by column chromatography on alumina using hexane as an eluent and following vacuum-transferred gave 1a as dark-red oil. The reaction conditions were not optimized. The characterization data of the diallylferrocene substrates are given below.

rac-1,1'-Diallyl-2,4-di(tert-butyl)ferrocene (1a). Yield: 42\%. ${ }^{1} \mathrm{H}$ NMR $\left(\mathrm{CDCl}_{3}\right): \delta 1.19(\mathrm{~s}, 9 \mathrm{H}), 1.28(\mathrm{~s}$, $9 \mathrm{H}), 3.09(\mathrm{~d}, \mathrm{~J}=6.6 \mathrm{~Hz}, 2 \mathrm{H}), 3.14(\mathrm{dd}, \mathrm{J}=16.0$ and $5.7 \mathrm{~Hz}, 1 \mathrm{H}), 3.25(\mathrm{dd}, \mathrm{J}=16.0$ and $6.8 \mathrm{~Hz}, 1 \mathrm{H}), 3.73$ (s, $1 \mathrm{H}), 3.76(\mathrm{~s}, 1 \mathrm{H}), 3.89(\mathrm{~s}, 1 \mathrm{H}), 3.92(\mathrm{~s}, 1 \mathrm{H}), 4.06(\mathrm{~s}, 1 \mathrm{H}), 4.13(\mathrm{~s}, 1 \mathrm{H}), 4.92-5.02(\mathrm{~m}, 4 \mathrm{H}), 5.86-5.96(\mathrm{~m}, 2 \mathrm{H})$. ${ }^{13} \mathrm{C}\left\{{ }^{1} \mathrm{H}\right\}$ NMR $\left(\mathrm{CDCl}_{3}\right): \delta 30.5,31.5,31.90,31.92,33.8,34.3,64.1,67.7,68.2,68.7,69.4,70.5,81.8,86.6,96.8$, 98.8, 114.6, 115.0, 138.3, 138.4. Anal. Calcd for $\mathrm{C}_{24} \mathrm{H}_{34} \mathrm{Fe}: \mathrm{C}, 76.18$; H, 9.06. Found: C, 76.06; H, 8.93. HRMS Calcd for $\mathrm{C}_{24} \mathrm{H}_{34} \mathrm{Fe}$ : 378.2008. Found: 378.2005. 
rac-1-Allyl-1'-((E)-2-butenyl)-2,4-di(tert-butyl)ferrocene (1b). Yield: 36\%. ${ }^{1} \mathrm{H} \mathrm{NMR}\left(\mathrm{CDCl}_{3}\right): \delta 1.19$ (s, 9H), $1.28(\mathrm{~s}, 9 \mathrm{H}), 1.63(\mathrm{dd}, \mathrm{J}=1.4$ and $6.4 \mathrm{~Hz}, 3 \mathrm{H}), 3.01(\mathrm{~d}, \mathrm{~J}=6.3 \mathrm{~Hz}, 2 \mathrm{H}), 3.09-3.28(\mathrm{~m}, 2 \mathrm{H}), 3.72(\mathrm{~s}, 1 \mathrm{H})$, $3.74(\mathrm{~s}, 1 \mathrm{H}), 3.87(\mathrm{~s}, 1 \mathrm{H}), 3.91(\mathrm{~s}, 1 \mathrm{H}), 4.04(\mathrm{~s}, 1 \mathrm{H}), 4.11(\mathrm{~s}, 1 \mathrm{H}), 4.98-5.02(\mathrm{~m}, 2 \mathrm{H}), 5.35-5.44(\mathrm{~m}, 1 \mathrm{H}), 5.49-$ $5.57(\mathrm{~m}, 1 \mathrm{H}), 5.87-5.97(\mathrm{~m}, 1 \mathrm{H}) .{ }^{13} \mathrm{C}\left\{{ }^{1} \mathrm{H}\right\} \mathrm{NMR}\left(\mathrm{CDCl}_{3}\right): \delta 17.8,30.5,31.5,31.9,32.4,32.5,34.3,64.0,67.6$, 68.0, 68.6, 69.3, 70.4, 81.7, 87.6, 96.8, 98.8, 114.9, 125.1, 131.0, 138.3. Anal. Calcd for $\mathrm{C}_{25} \mathrm{H}_{36} \mathrm{Fe}: \mathrm{C}, 76.52 ; \mathrm{H}$, 9.25. Found: $\mathrm{C}, 76.46 ; \mathrm{H}, 9.36$. HRMS Calcd for $\mathrm{C}_{25} \mathrm{H}_{36} \mathrm{Fe}: 392.2165$. Found: 392.2164.

rac-1-Allyl-1'-((E)-3-phenylallyl)-2,4-di(tert-butyl)ferrocene (1c). Yield: 42\%. ${ }^{1} \mathrm{H} \mathrm{NMR}\left(\mathrm{CDCl}_{3}\right): \delta 1.20$ (s, 9H), $1.29(\mathrm{~s}, 9 \mathrm{H}), 3.15-3.31(\mathrm{~m}, 2 \mathrm{H}), 3.26(\mathrm{~d}, \mathrm{~J}=5.4 \mathrm{~Hz}, 2 \mathrm{H}), 3.75-3.76(\mathrm{~m}, 2 \mathrm{H}), 3.92-3.94(\mathrm{~m}, 2 \mathrm{H}), 4.05-$ $4.14(\mathrm{~m}, 2 \mathrm{H}), 4.97-5.03(\mathrm{~m}, 2 \mathrm{H})$, 5.88-5.98 (m, 1H), 6.25-6.36 (m, 2H), 7.15-7.20 (m, 1H), 7.25-7.34 (m, 4H). ${ }^{13} \mathrm{C}\left\{{ }^{1} \mathrm{H}\right\}$ NMR $\left(\mathrm{CDCl}_{3}\right): \delta 30.5,31.6,32.0,32.4,33.0,34.3,64.0,67.7,68.2,68.7,69.4,70.5,81.8,86.6,96.8$, 98.8, 115.0, 126.1, 126.9, 128.5, 129.9, 130.1, 137.6, 138.3. Anal. Calcd for $\mathrm{C}_{30} \mathrm{H}_{38} \mathrm{Fe}: \mathrm{C}, 79.28 ; \mathrm{H}, 8.43$. Found: C, 79.16; $\mathrm{H}, 8.24$. HRMS Calcd for $\mathrm{C}_{30} \mathrm{H}_{38} \mathrm{Fe}: 454.2321$. Found: 454.2325.

rac-1-Allyl-1'-(3-methyl-2-butenyl)-2,4-di(tert-butyl)ferrocene (1d). Yield: $33 \% .{ }^{1} \mathrm{H} \mathrm{NMR}\left(\mathrm{CDCl}_{3}\right): \delta$ $1.20(\mathrm{~s}, 9 \mathrm{H}), 1.29(\mathrm{~s}, 9 \mathrm{H}), 1.66(\mathrm{~s}, 3 \mathrm{H}), 1.68(\mathrm{~s}, 3 \mathrm{H}), 3.02(\mathrm{~d}, \mathrm{~J}=7.2 \mathrm{~Hz}, 2 \mathrm{H}), 3.16(\mathrm{dd}, \mathrm{J}=5.9$ and $16.0 \mathrm{~Hz}, 1 \mathrm{H})$, $3.27(\mathrm{dd}, \mathrm{J}=6.7$ and $16.0 \mathrm{~Hz}, 1 \mathrm{H}), 3.71(\mathrm{br}, 1 \mathrm{H}), 3.72(\mathrm{br}, 1 \mathrm{H}), 3.86(\mathrm{br}, 1 \mathrm{H}), 3.89(\mathrm{br}, 1 \mathrm{H}), 4.02(\mathrm{br}, 1 \mathrm{H}), 4.09$ (br, 1H), $4.98(\mathrm{br}, 1 \mathrm{H}), 5.01-5.02(\mathrm{~m}, 1 \mathrm{H}), 5.24-5.28(\mathrm{~m}, 1 \mathrm{H}), 5.89-5.96(\mathrm{~m}, 1 \mathrm{H}) .{ }^{13} \mathrm{C}\left\{{ }^{1} \mathrm{H}\right\} \mathrm{NMR}\left(\mathrm{CDCl}_{3}\right): \delta$ 17.8, 25.7, 27.8, 30.5, 31.5, 31.9, 32.4, 34.3, 63.8, 67.6, 67.9, 68.4, 69.1, 70.2, 81.7, 88.4, 96.7, 98.7, 114.9, 124.3, 131.1, 138.4. Anal. Calcd for $\mathrm{C}_{26} \mathrm{H}_{38} \mathrm{Fe}$ : C, 76.84; H, 9.42. Found: C, 76.70; H, 9.59. HRMS Calcd for $\mathrm{C}_{26} \mathrm{H}_{38} \mathrm{Fe}: 406.2321$. Found: 406.2328 .

rac-1-Allyl-1'-(2-methylallyl)-2,4-di(tert-butyl)ferrocene (1e). Yield: $36 \% .{ }^{1} \mathrm{H}$ NMR $\left(\mathrm{CDCl}_{3}\right): \delta 1.18$ (s, 9H), $1.27(\mathrm{~s}, 9 \mathrm{H}), 1.65(\mathrm{~s}, 3 \mathrm{H}), 3.01(\mathrm{br}, 2 \mathrm{H}), 3.07-3.12(\mathrm{~m}, 1 \mathrm{H}), 3.22(\mathrm{dd}, \mathrm{J}=6.7$ and $15.8 \mathrm{~Hz}, 1 \mathrm{H}), 3.72(\mathrm{br}$, 1H), 3.76 (br, 1H), 3.93 (br, 2H), $4.08(\mathrm{br}, 1 \mathrm{H}), 4.13(\mathrm{br}, 1 \mathrm{H}), 4.59$ (s, 1H), $4.62(\mathrm{~s}, 1 \mathrm{H}), 4.99(\mathrm{~s}, 1 \mathrm{H}), 5.03(\mathrm{~d}, \mathrm{~J}$ $=4.8 \mathrm{~Hz}, 1 \mathrm{H}), 5.88-5.97(\mathrm{~m}, 1 \mathrm{H}) .{ }^{13} \mathrm{C}\left\{{ }^{1} \mathrm{H}\right\} \mathrm{NMR}\left(\mathrm{CDCl}_{3}\right): \delta 22.2,30.5,31.5,31.9,32.4,34.3,38.5,64.1,67.7$, 68.1, 68.8, 70.1, 71.2, 81.7, 86.0, 96.8, 98.8, 110.8, 115.0, 138.2, 146.5. Anal. Calcd for $\mathrm{C}_{25} \mathrm{H}_{36} \mathrm{Fe}$ : C, 76.52; $\mathrm{H}$, 9.25. Found: C, 76.30; H, 9.03. HRMS Calcd for $\mathrm{C}_{25} \mathrm{H}_{36} \mathrm{Fe}: 392.2165$. Found: 392.2165.

rac-1-Allyl-1'-(2-methylallyl)-2,4-dicyclohexylferrocene (1f). Yield: 30\%. ${ }^{1} \mathrm{H}$ NMR $\left(\mathrm{CDCl}_{3}\right): \delta 0.91$ (dq, $\mathrm{J}=2.4$ and $12.3 \mathrm{~Hz}, 1 \mathrm{H}), 1.15-1.40(\mathrm{~m}, 9 \mathrm{H}), 1.61-1.78(\mathrm{~m}, 6 \mathrm{H}), 1.66(\mathrm{~s}, 3 \mathrm{H}), 1.84-1.93(\mathrm{~m}, 3 \mathrm{H}), 2.13-2.25(\mathrm{~m}$, $3 \mathrm{H}), 2.94-3.09(\mathrm{~m}, 4 \mathrm{H}), 3.77-3.97(\mathrm{~m}, 6 \mathrm{H}), 4.58(\mathrm{br}, 1 \mathrm{H}), 4.63(\mathrm{br}, 1 \mathrm{H}), 4.99-5.06(\mathrm{~m}, 2 \mathrm{H}), 5.91-6.01(\mathrm{~m}, 1 \mathrm{H})$. ${ }^{13} \mathrm{C}\left\{{ }^{1} \mathrm{H}\right\}$ NMR $\left(\mathrm{CDCl}_{3}\right): \delta 22.3,26.5,26.6,26.8(2 \mathrm{C}), 26.9,27.0,32.26,32.29,34.3,34.4,36.3,36.4,37.4,38.1$, 64.0, 66.9, 69.0, 69.1, 70.5, 71.0, 82.9, 86.2, 92.5, 92.9, 110.1, 115.0, 138.0, 146.5. Anal. Calcd for $\mathrm{C}_{29} \mathrm{H}_{40} \mathrm{Fe}$ : C, 78.36; H, 9.07. Found: C, 78.47; H, 9.20. HRMS Calcd for $\mathrm{C}_{29} \mathrm{H}_{40} \mathrm{Fe}: 444.2477$. Found: 444.2484.

rac-1-Allyl-1'-(2-methylallyl)-2,4-bis(trimethylsilyl)ferrocene (1g). Yield: 14\%. ${ }^{1} \mathrm{H}$ NMR $\left(\mathrm{CDCl}_{3}\right): \delta$ $0.22(\mathrm{~s}, 9 \mathrm{H}), 0.26(\mathrm{~s}, 9 \mathrm{H}), 1.64(\mathrm{~s}, 3 \mathrm{H}), 3.00(\mathrm{br}, 2 \mathrm{H}), 3.13(\mathrm{~d}, \mathrm{~J}=6.2 \mathrm{~Hz}, 2 \mathrm{H}), 3.79(\mathrm{~s}, 1 \mathrm{H}), 3.88(\mathrm{~s}, 1 \mathrm{H}), 3.90$ (s, $1 \mathrm{H}), 3.96(\mathrm{~s}, 1 \mathrm{H}), 4.02(\mathrm{~s}, 1 \mathrm{H}), 4.07(\mathrm{~s}, 1 \mathrm{H}), 4.58(\mathrm{~s}, 1 \mathrm{H}), 4.62(\mathrm{~s}, 1 \mathrm{H}), 4.98(\mathrm{~s}, 1 \mathrm{H}), 5.01(\mathrm{~d}, \mathrm{~J}=3.9 \mathrm{~Hz}, 1 \mathrm{H})$, 5.87-5.97 $(\mathrm{m}, 1 \mathrm{H}) . \quad{ }^{13} \mathrm{C}\left\{{ }^{1} \mathrm{H}\right\}$ NMR $\left(\mathrm{CDCl}_{3}\right): \delta 0.06,0.6,22.2,34.2,38.4,68.1,68.4,69.7,70.8,73.3,74.0,78.2$, 79.4, 86.5, 94.4, 110.3, 115.0, 138.2, 146.2. Anal. Calcd for $\mathrm{C}_{23} \mathrm{H}_{36} \mathrm{FeSi}_{2}$ : C, 65.07; H, 8.55. Found: C, 65.13; $\mathrm{H}$, 8.43. HRMS Calcd for $\mathrm{C}_{23} \mathrm{H}_{36} \mathrm{FeSi}_{2}$ : 424.1703. Found: 424.1702.

Kinetic Resolution of Racemic 1 by Molybdenum-Catalyzed Asymmetric Ring-Closing Metathesis Reaction. The reaction conditions and the results are summarized in Table 1 of the main text. A typical procedure is given for the reaction of entry 6: a THF solution of $\mathrm{Mo}\left(=\mathrm{NC}_{6} \mathrm{H}_{3}-2,6-{ }^{i} \mathrm{Pr}_{2}\right)$ $\left(=\mathrm{CHCMe}_{2} \mathrm{Ph}\right)(\mathrm{OTf})_{2}(\mathrm{dme})(0.1 \mathrm{M}, 100 \mu \mathrm{L}, 10 \mu \mathrm{mol})$ and a THF solution of 3,3'- ${ }^{t} \mathrm{Bu}_{2}-\mathrm{H}_{8}$-binaphthol $\mathrm{K}_{2}$ salt $(0.1$ $\mathrm{M}, 100 \mu \mathrm{L}, 10 \mu \mathrm{mol}$ ) was mixed at $-30^{\circ} \mathrm{C}$, and then the mixture was stirred at room temperature for $1 \mathrm{~h}$. To this was added $1 \mathrm{e}(39.3 \mathrm{mg}, 100 \mu \mathrm{mol})$ and benzene $(20 \mathrm{~mL})$ and the solution was stirred at $50{ }^{\circ} \mathrm{C}$ for $24 \mathrm{~h}$. After quenching by addition of acetone (ca. $100 \mu \mathrm{L}$ ), the reaction mixture was passed through a pad of silica gel using hexane/ $\mathrm{Et}_{2} \mathrm{O}(9 / 1)$ as an eluent. The solvent was removed under reduced pressure and the residue was 
purified by preparative HPLC (LC-908 recycle HPLC system (Japan Analytical Industry Co. Ltd.) with a GPC column (JAIGEL-H, chloroform, $3.5 \mathrm{~mL} / \mathrm{min})$ ) to give the RCM product $\mathbf{2 e}(16.8 \mathrm{mg}, 46 \%)$ and the recovered 1e $(18.5 \mathrm{mg}, 47 \%)$. The recovered $1 \mathrm{e}$ was quantitatively converted to $2 \mathrm{e}$ by a treatment with the $2 \mathrm{nd}-$ generation Grubbs' catalyst ( $2 \mathrm{~mol} \%$ ) in refluxing $\mathrm{CH}_{2} \mathrm{Cl}_{2}$ for a chiral HPLC analysis. The characterization data of the RCM products and the conditions for chiral HPLC analysis are described below.

1,1'-(2-Buten-1,4-diyl)-2,4-di(tert-butyl)ferrocene (2a). ${ }^{1} \mathrm{H}$ NMR $\left(\mathrm{CDCl}_{3}\right): \delta 1.15$ (s, 9H), 1.31 (s, 9H), $2.74(\mathrm{dd}, \mathrm{J}=7.3$ and $14.6 \mathrm{~Hz}, 1 \mathrm{H}), 2.88(\mathrm{dd}, \mathrm{J}=7.3$ and $14.6 \mathrm{~Hz}, 1 \mathrm{H}), 3.02(\mathrm{dd}, \mathrm{J}=6.0$ and $15.1 \mathrm{~Hz}, 1 \mathrm{H}), 3.48$ $(\mathrm{dd}, \mathrm{J}=6.0$ and $15.1 \mathrm{~Hz}, 1 \mathrm{H}), 3.79(\mathrm{~s}, 1 \mathrm{H}), 3.80(\mathrm{~s}, 1 \mathrm{H}), 3.93(\mathrm{~s}, 1 \mathrm{H}), 3.98-3.99(\mathrm{~m}, 2 \mathrm{H}), 4.13(\mathrm{~s}, 1 \mathrm{H}), 5.93-6.02$ $(\mathrm{m}, 1 \mathrm{H}) .{ }^{13} \mathrm{C}\left\{{ }^{1} \mathrm{H}\right\}$ NMR $\left(\mathrm{CDCl}_{3}\right): \delta 24.1,24.3,30.4,31.4,32.0,32.6,63.3,65.7,66.3,66.9,70.1,70.6,82.0$, 86.2, 97.6, 99.6, 130.2, 131.6. Anal. Calcd for $\mathrm{C}_{22} \mathrm{H}_{30} \mathrm{Fe}$ : C, 75.43; H, 8.63. Found: C, 75.29; H, 8.77. HRMS Calcd for $\mathrm{C}_{22} \mathrm{H}_{30} \mathrm{Fe}: 350.1695$. Found: 350.1697. $[\alpha]^{31}{ }_{\mathrm{D}}=-9.2\left(c 0.56, \mathrm{CHCl}_{3}\right.$ for the sample of $(R)-66 \%$ ee $)$. Chiral HPLC Analysis Conditions: Chiralcel OD-H $\times 2$; eluent: hexane $/ \mathrm{PrOH}=1000 / 1$; flow rate: $0.5 \mathrm{~mL} / \mathrm{min}$; $\mathrm{t}_{1}=32.1 \min (R$-isomer $), \mathrm{t}_{2}=34.8 \min (S$-isomer $)$.

1,1'-(3-Methyl-2-buten-1,4-diyl)-2,4-di(tert-butyl)ferrocene (2e). ${ }^{1} \mathrm{H}$ NMR $\left(\mathrm{CDCl}_{3}\right): \delta 1.14(\mathrm{~s}, 9 \mathrm{H}), 1.28$ (s, 9H), $1.93(\mathrm{~s}, 3 \mathrm{H}), 2.59-2.67(\mathrm{~m}, 2 \mathrm{H}), 3.12(\mathrm{~d}, \mathrm{~J}=14.7 \mathrm{~Hz}, 1 \mathrm{H}), 3.28(\mathrm{dd}, \mathrm{J}=7.4$ and $14.7 \mathrm{~Hz}, 1 \mathrm{H}), 3.73(\mathrm{~s}$, $1 \mathrm{H}), 3.82(\mathrm{~s}, 1 \mathrm{H}), 3.89(\mathrm{~s}, 1 \mathrm{H}), 3.99(\mathrm{~s}, 1 \mathrm{H}), 4.02(\mathrm{~s}, 1 \mathrm{H}), 4.14(\mathrm{~s}, 1 \mathrm{H}), 5.67(\mathrm{t}, \mathrm{J}=7.4 \mathrm{~Hz}, 1 \mathrm{H}) . \quad{ }^{13} \mathrm{C}\left\{{ }^{1} \mathrm{H}\right\} \mathrm{NMR}$ $\left(\mathrm{CDCl}_{3}\right): \delta$ 24.8, 26.7, 29.4, 30.4, 31.4, 32.1, 32.6, 63.6, 66.0, 66.1, 66.8, 70.3, 70.6, 83.1, 85.3, 98.3, 99.9, 124.1, 138.1. Anal. Calcd for $\mathrm{C}_{23} \mathrm{H}_{32} \mathrm{Fe}: \mathrm{C}, 75.82 ; \mathrm{H}, 8.85$. Found: $\mathrm{C}, 76.08 ; \mathrm{H}, 8.91$. HRMS Calcd for $\mathrm{C}_{23} \mathrm{H}_{32} \mathrm{Fe}$ : 364.1852. Found: 364.1856. $[\alpha]_{\mathrm{D}}^{30}=+22\left(c 0.50, \mathrm{CHCl}_{3}\right.$ for the sample of $(R)-99.5 \%$ ee $)$. Chiral HPLC Analysis Conditions: Chiralcel OD-H; eluent: hexane $/ 2 \mathrm{PrOH}=1000 / 1$; flow rate: $0.2 \mathrm{~mL} / \mathrm{min} ; \mathrm{t}_{1}=18.4 \mathrm{~min}(R-$ isomer), $\mathrm{t}_{2}=20.3 \mathrm{~min}(S$-isomer $)$.

1,1'-(3-Methyl-2-buten-1,4-diyl)-2,4-dicyclohexylferrocene (2f). ${ }^{1} \mathrm{H}$ NMR $\left(\mathrm{CDCl}_{3}\right): \delta$ 0.87-0.97 (m, 1H), 1.08-1.40 (m, 9H), 1.66-1.75 (m, 6H), 1.83-1.89 (m, 3H), $1.93(\mathrm{~s}, 3 \mathrm{H}), 1.97-2.10(\mathrm{~m}, 2 \mathrm{H}), 2.33-2.39(\mathrm{~m}, 1 \mathrm{H})$, $2.67(\mathrm{dd}, \mathrm{J}=7.8$ and $14.6 \mathrm{~Hz}, 1 \mathrm{H}), 2.78(\mathrm{~d}, \mathrm{~J}=14.4 \mathrm{~Hz}, 1 \mathrm{H}), 2.91(\mathrm{dd}, \mathrm{J}=7.8$ and $14.6 \mathrm{~Hz}, 1 \mathrm{H}), 3.02(\mathrm{~d}, \mathrm{~J}=$ $14.4 \mathrm{~Hz}, 1 \mathrm{H}), 3.53(\mathrm{br}, 1 \mathrm{H}), 3.79(\mathrm{br}, 1 \mathrm{H}), 3.85(\mathrm{br}, 1 \mathrm{H}), 3.87(\mathrm{br}, 1 \mathrm{H}), 3.94(\mathrm{br}, 1 \mathrm{H}), 4.09(\mathrm{br}, 1 \mathrm{H}), 5.72(\mathrm{t}, \mathrm{J}=$ $7.8 \mathrm{~Hz}, 1 \mathrm{H}) .{ }^{13} \mathrm{C}\left\{{ }^{1} \mathrm{H}\right\} \mathrm{NMR}\left(\mathrm{CDCl}_{3}\right): \delta 14.3,22.8,23.6,26.65,26.74,26.8,27.1,29.6,31.5,31.7,34.4,34.5$, $36.3,37.25,37.33,63.2,66.5,66.6,67.6,70.3,71.9,84.9,86.3,93.0,93.8,123.6,138.1$. Anal. Calcd for $\mathrm{C}_{27} \mathrm{H}_{36} \mathrm{Fe}: \mathrm{C}, 77.88 ; \mathrm{H}, 8.71$. Found: $\mathrm{C}, 77.70 ; \mathrm{H}, 8.92$. HRMS Calcd for $\mathrm{C}_{27} \mathrm{H}_{36} \mathrm{Fe}: 416.2165$. Found: 416.2166. $[\alpha]^{30}=-23\left(c 1.3, \mathrm{CHCl}_{3}\right.$ for the sample of $(R)-82 \%$ ee $)$. Chiral HPLC Analysis Conditions: Chiralcel OD-H $\times 2$; eluent: hexane $/{ }^{i} \mathrm{PrOH}=1000 / 1$; flow rate: $0.4 \mathrm{~mL} / \mathrm{min} ; \mathrm{t}_{1}=21.4 \mathrm{~min}(S$-isomer $), \mathrm{t}_{2}=27.6 \mathrm{~min}(R$-isomer $)$.

1,1'-(3-Methyl-2-buten-1,4-diyl)-2,4-bis(trimethylsilyl)ferrocene (2g). ${ }^{1} \mathrm{H}$ NMR $\left(\mathrm{CDCl}_{3}\right): \delta 0.17(\mathrm{~s}, 9 \mathrm{H})$, $0.27(\mathrm{~s}, 9 \mathrm{H}), 1.94(\mathrm{~s}, 3 \mathrm{H}), 2.69(\mathrm{~d}, \mathrm{~J}=14.4 \mathrm{~Hz}, 1 \mathrm{H}), 2.75(\mathrm{dd}, \mathrm{J}=8.2$ and $14.8 \mathrm{~Hz}, 1 \mathrm{H}), 3.02(\mathrm{dd}, \mathrm{J}=7.3$ and $14.8 \mathrm{~Hz}, 1 \mathrm{H}), 3.10(\mathrm{~d}, \mathrm{~J}=14.4 \mathrm{~Hz}, 1 \mathrm{H}), 3.84(\mathrm{~s}, 1 \mathrm{H}), 3.87(\mathrm{~s}, 1 \mathrm{H}), 3.92(\mathrm{~s}, 1 \mathrm{H}), 3.97(\mathrm{~s}, 1 \mathrm{H}), 4.02(\mathrm{~s}, 1 \mathrm{H}), 4.10$ $(\mathrm{s}, 1 \mathrm{H}), 5.76(\mathrm{t}, \mathrm{J}=7.8 \mathrm{~Hz}, 1 \mathrm{H}) . \quad{ }^{13} \mathrm{C}\left\{{ }^{1} \mathrm{H}\right\} \mathrm{NMR}\left(\mathrm{CDCl}_{3}\right): \delta-0.05,0.9,25.0,26.7,29.4,66.4,66.5,69.7,70.1$, 74.7, 74.8, 76.2, 78.5, 86.1, 96.3, 124.1, 138.1. Anal. Calcd for $\mathrm{C}_{21} \mathrm{H}_{32} \mathrm{FeSi}_{2}: \mathrm{C}, 63.61 ; \mathrm{H}, 8.13$. Found: C, 63.46; H, 8.04. HRMS Calcd for $\mathrm{C}_{21} \mathrm{H}_{32} \mathrm{FeSi}_{2}: 396.1390$. Found: 396.1395. $[\alpha]_{\mathrm{D}}^{31}=+50\left(c 2.0, \mathrm{CHCl}_{3}\right.$ for the sample of $(R)-97 \%$ ee). Chiral HPLC Analysis Conditions: Chiralcel OD-H $\times 2$; eluent: hexane $/ \mathrm{PrOH}=$ 2000/1; flow rate: $0.5 \mathrm{~mL} / \mathrm{min} ; \mathrm{t}_{1}=39.8 \mathrm{~min}(R$-isomer $), \mathrm{t}_{2}=42.5 \mathrm{~min}(S$-isomer $)$.

\section{References.}

(1) Panda, T. K.; Gamer, M. T.; Roesky, P. W. Organometallics 2003, 22, 877.

(2) Venier, C. G.; Casserly, E. W. J. Am. Chem. Soc. 1990, 112, 2808.

(3) Clark, T. J.; Killian, C. M.; Luthra, S.; Nile, T. A. J. Organomet. Chem. 1993, 462, 247.

(4) Ustynyuk, Y. A.; Kisin, A. V.; Pribytkova, I. M.; Zenkin, A. A.; Antonova, N. D. J. Organomet. Chem. 1972, 42, 47.

(5) Bunel, E. E.; Valle, L.; Manriquez, J. M. Organometallics 1985, 4, 1680. 
(6) Schrock, R. R.; Murdzek, J. S.; Bazan, G. C.; Robbins, J.; DiMare, M.; O'Regan, M. J. Am. Chem. Soc. 1990, 112, 3875 .

(7) Aeilts, S. L.; Cefalo, D. R.; Bonitatebus, Jr., P. J.; Houser, J. H.; Hoveyda, A. H.; Schrock, R. R. Angew. Chem. Int. Ed. 2001, 40, 1452.

(8) Trnka, T. M.; Morgan, J. P.; Sanford, M. S.; Wilhelm, T. E.; Scholl, M.; Choi, T.-L.; Ding, S.; Day, M. W.; Grubbs, R. H. J. Am. Chem. Soc. 2003, 125, 2546.

(9) Erker, G.; Aul, R. Chem. Ber. 1991, 1301.

(10) Manríquez, J. M.; Bunel, E. E.; Oelckers, B. Inorg. Synth. 1997, 31, 214. 

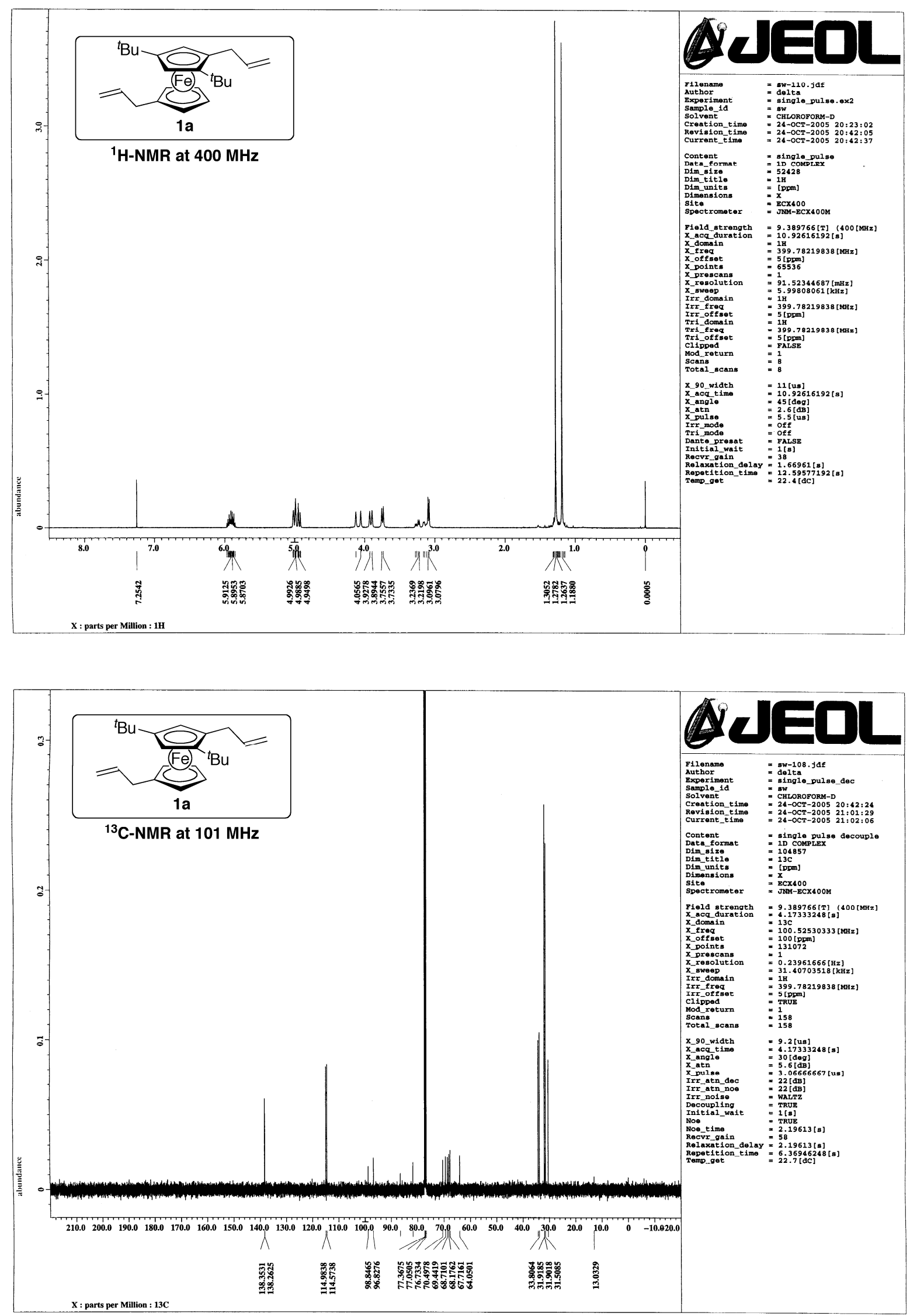

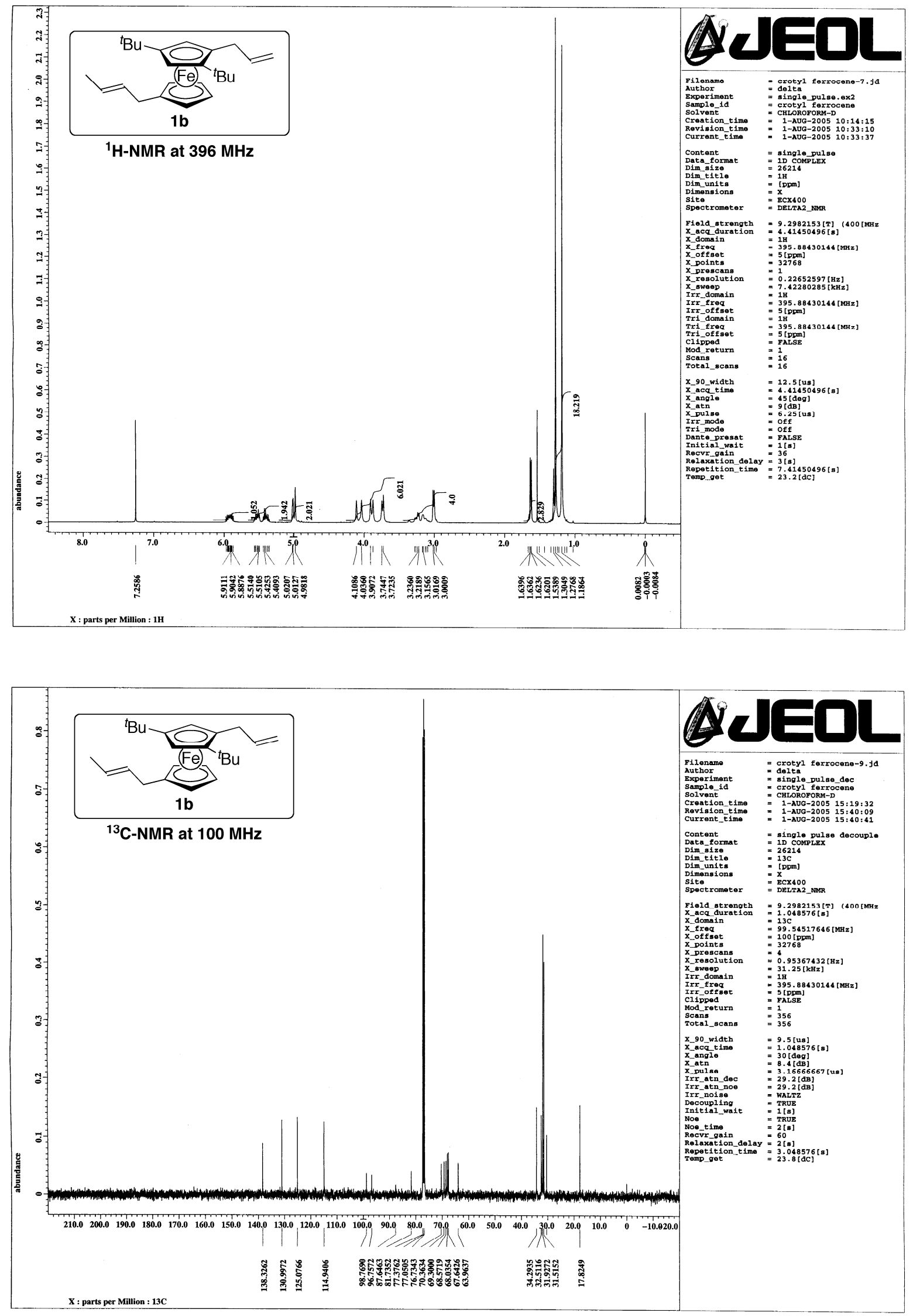

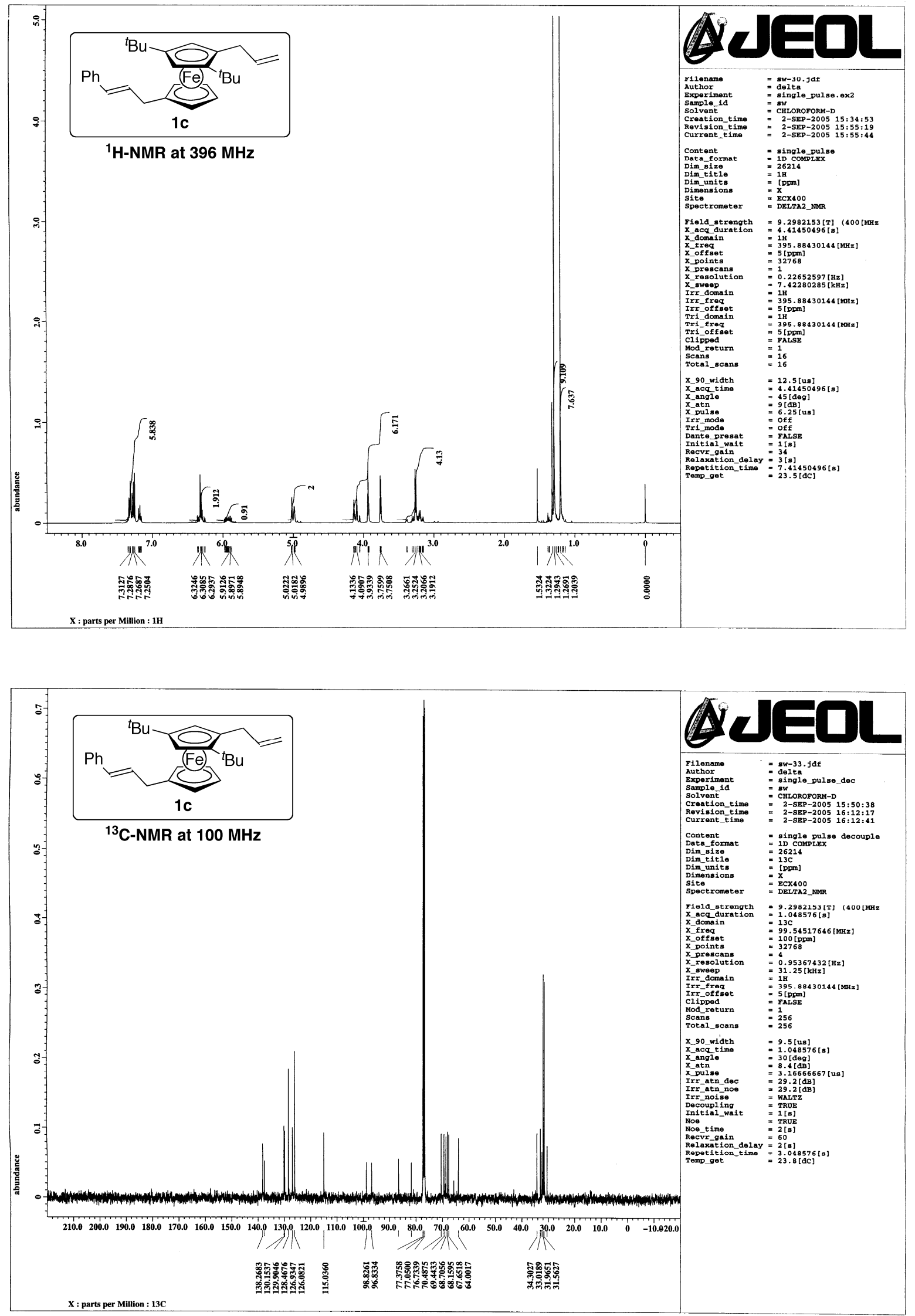

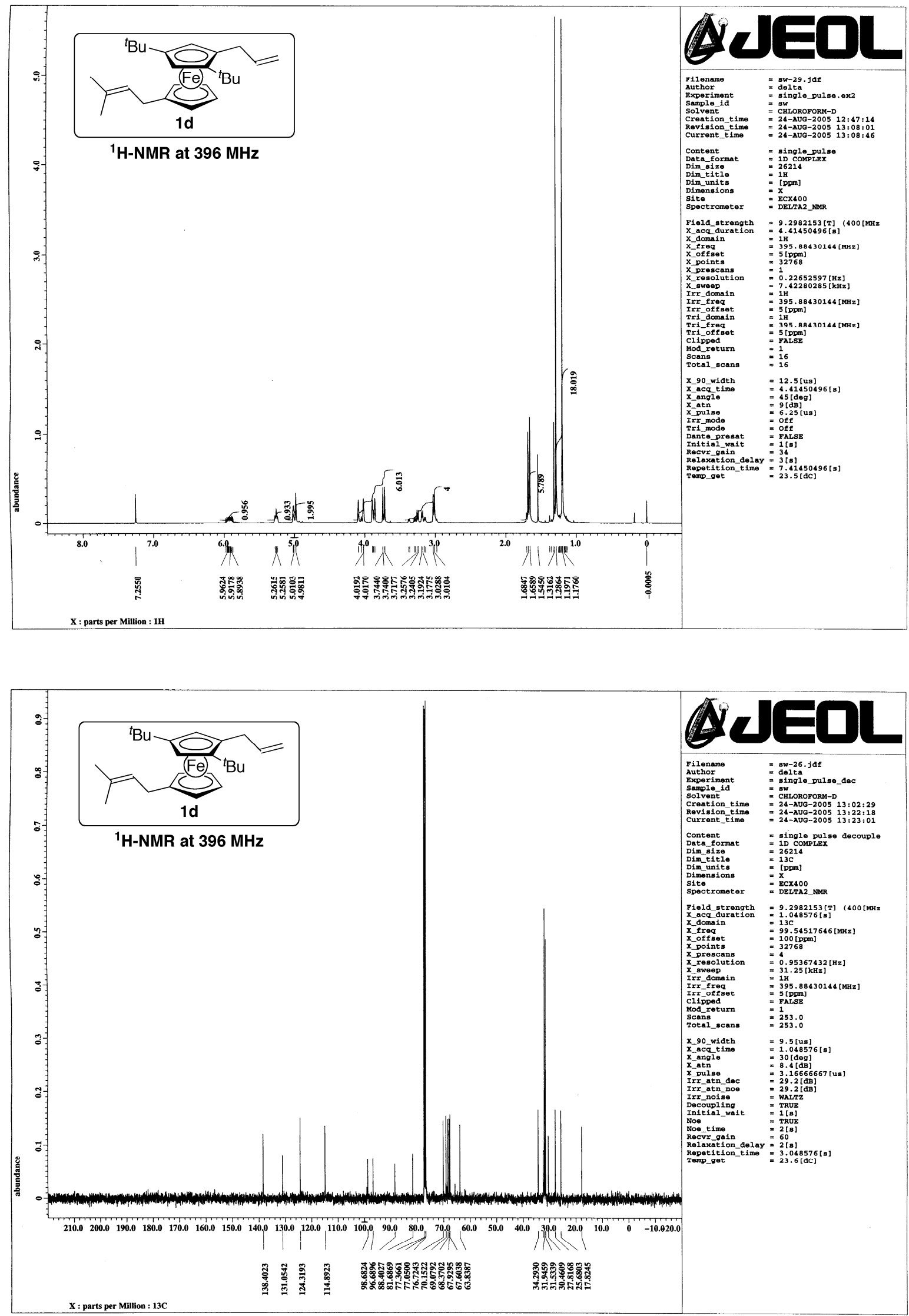

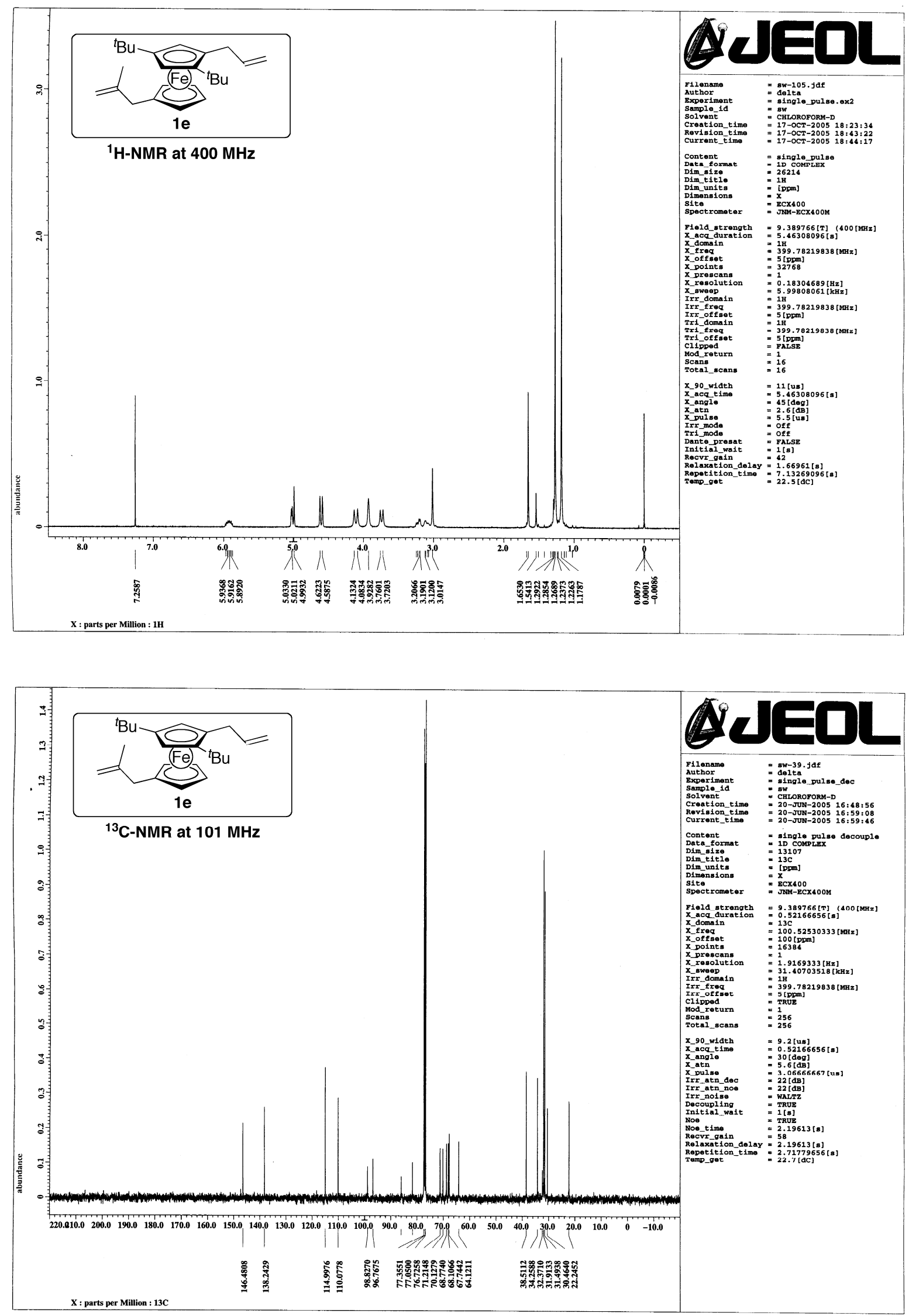

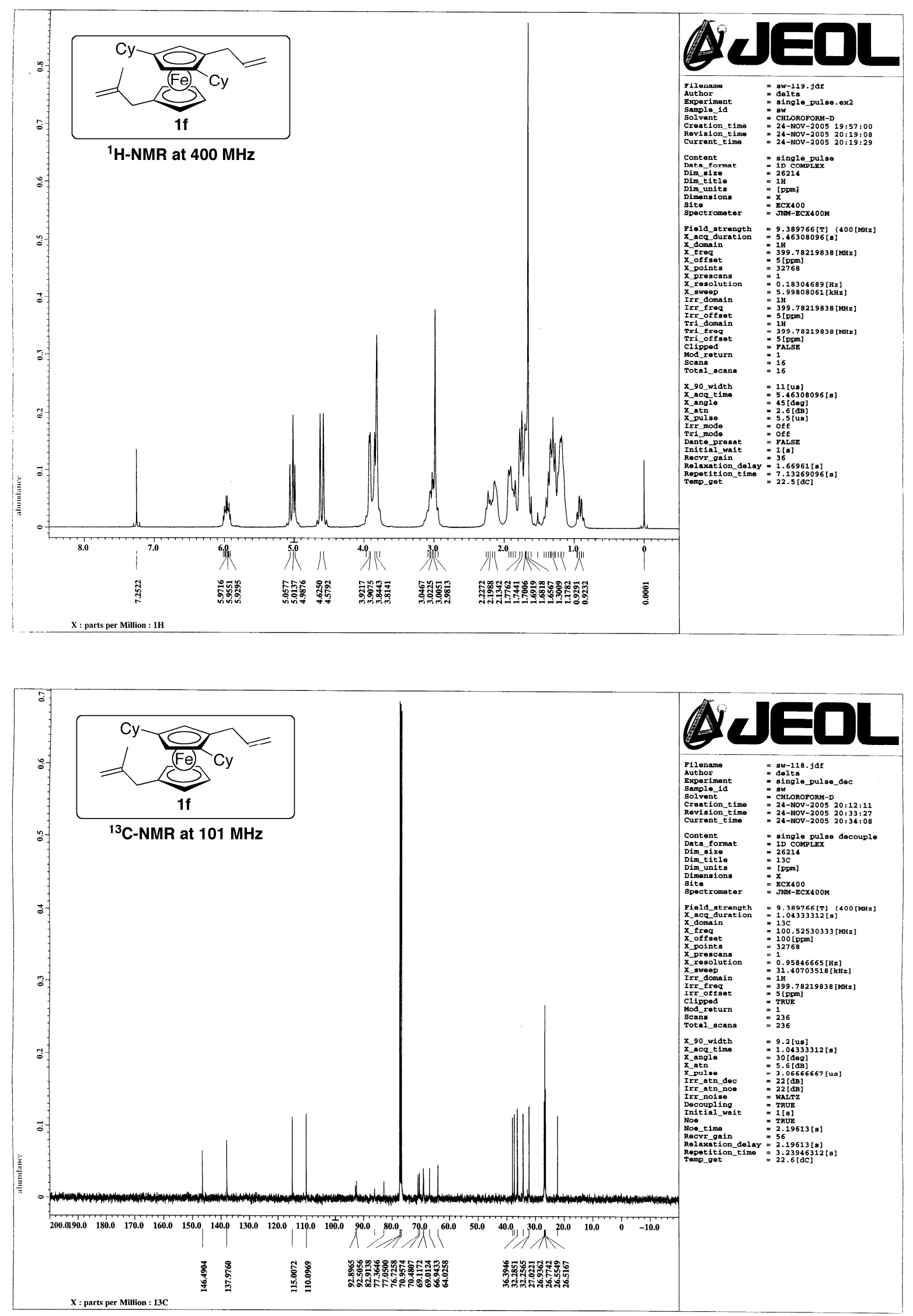

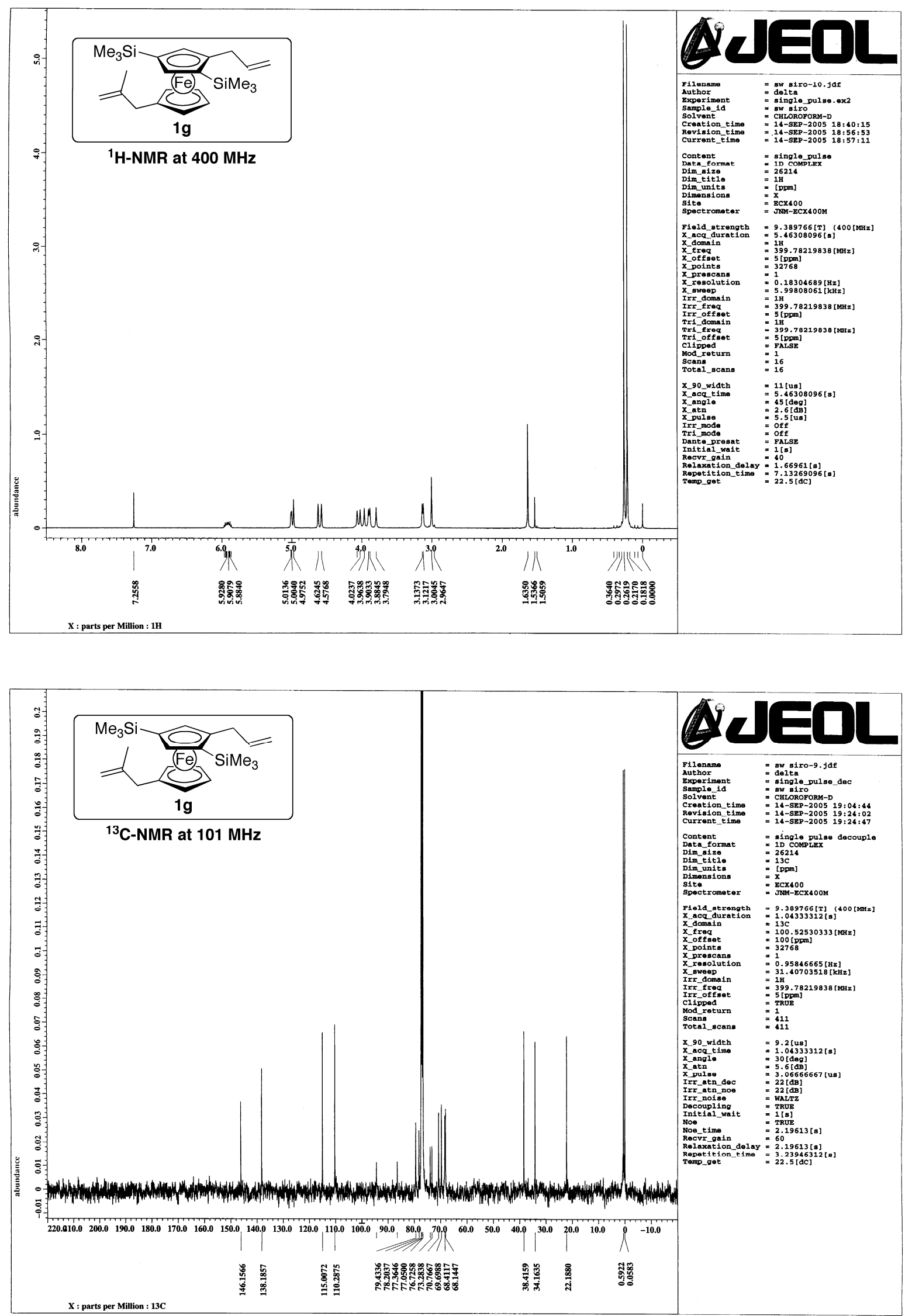

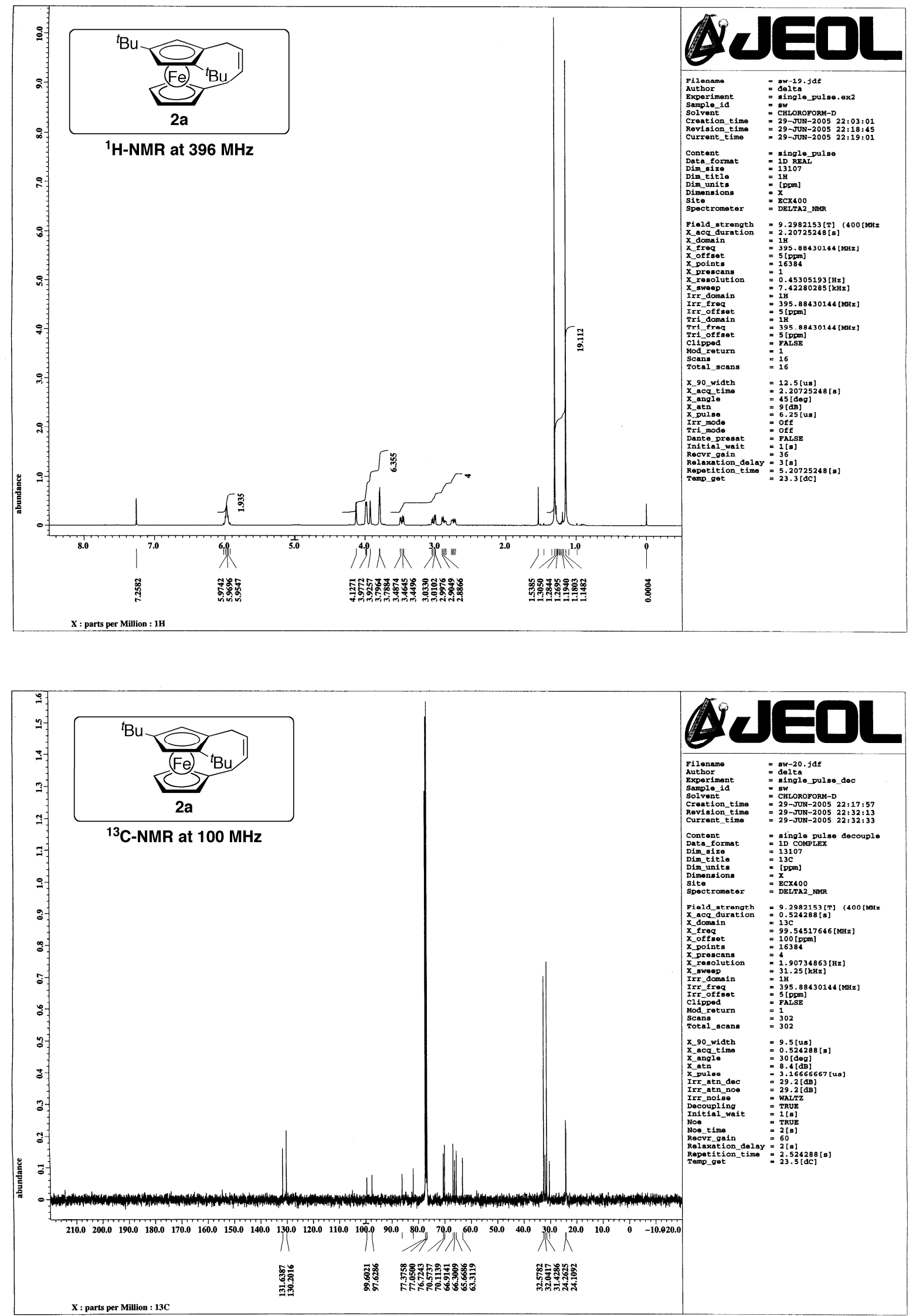

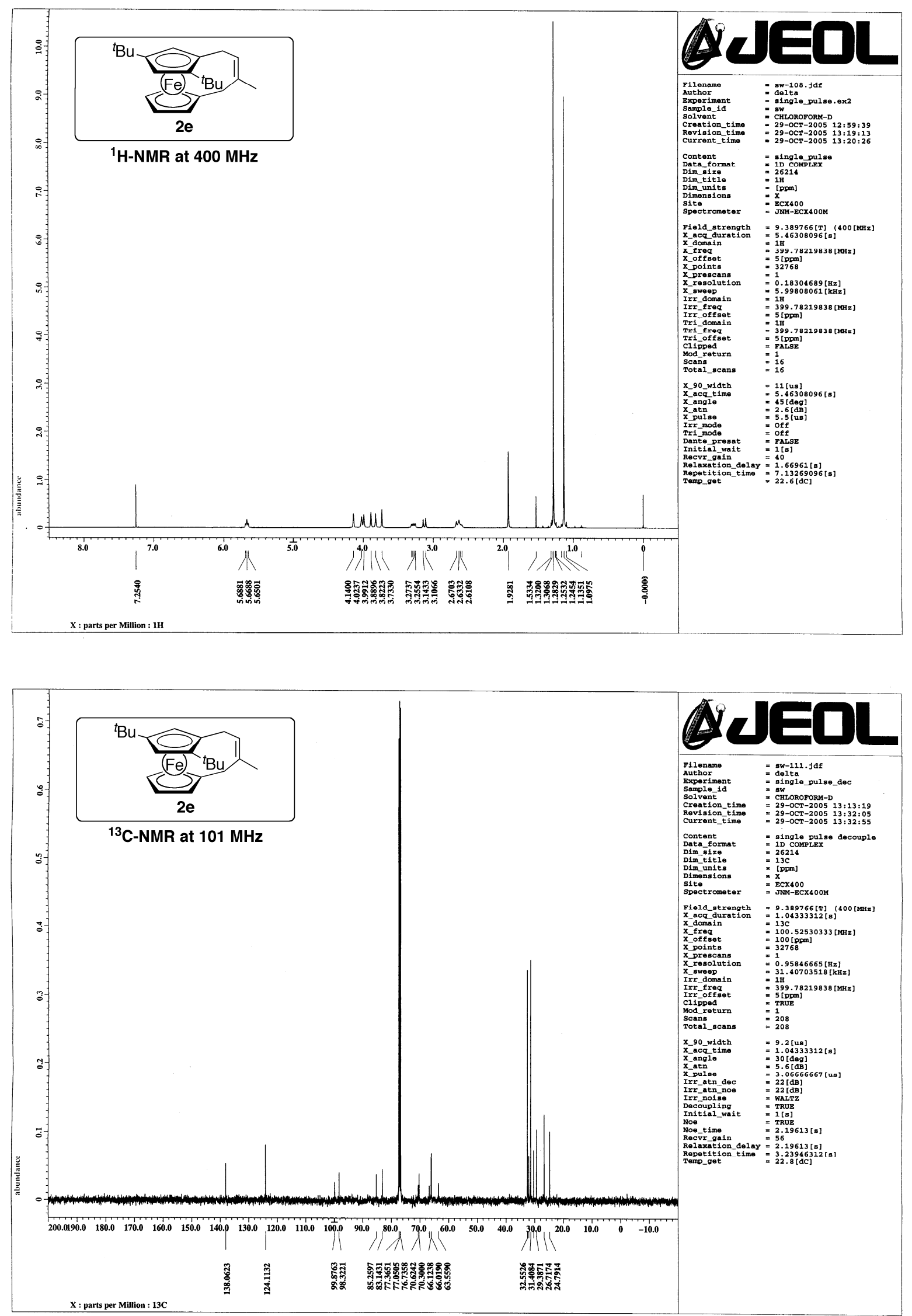

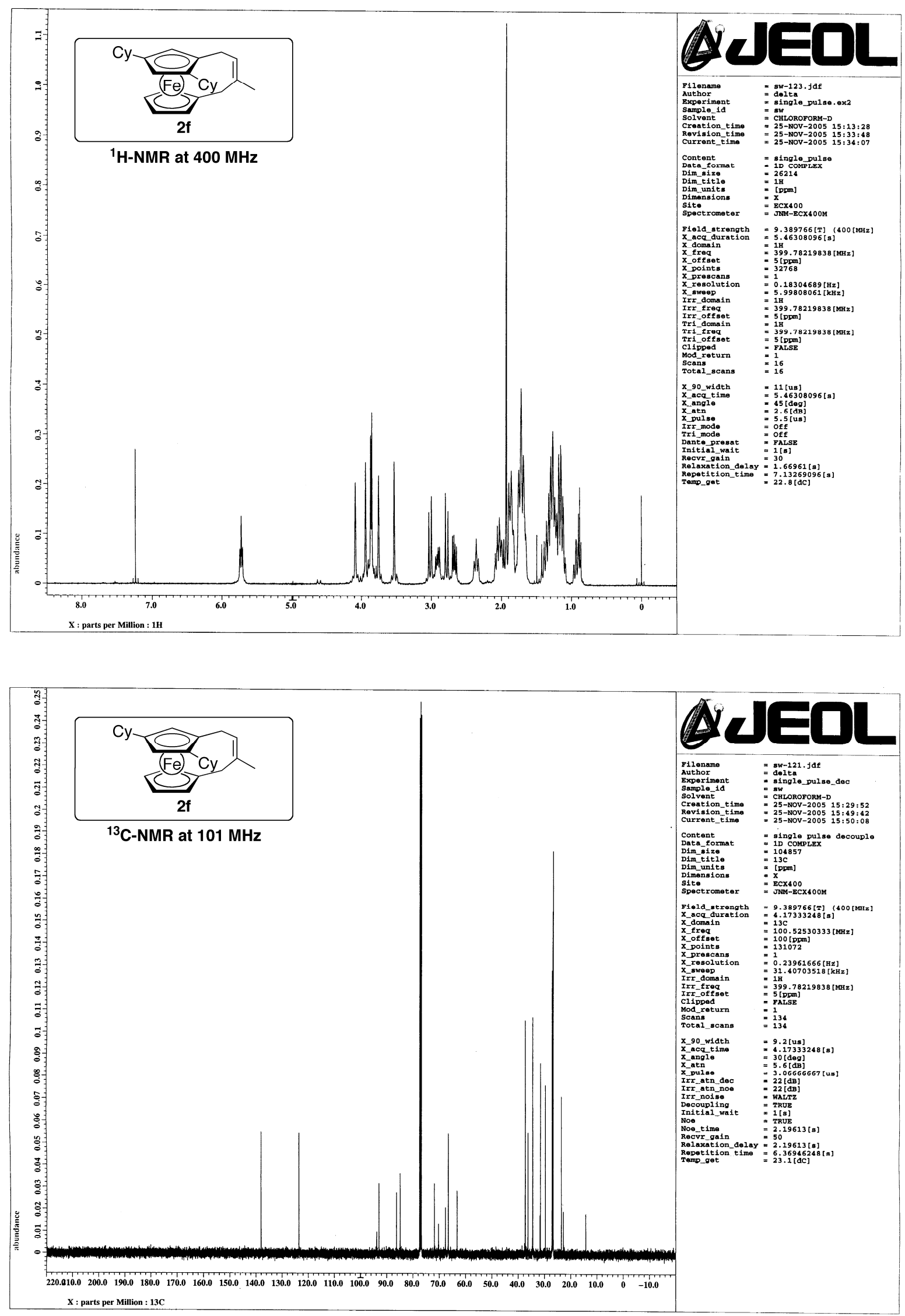

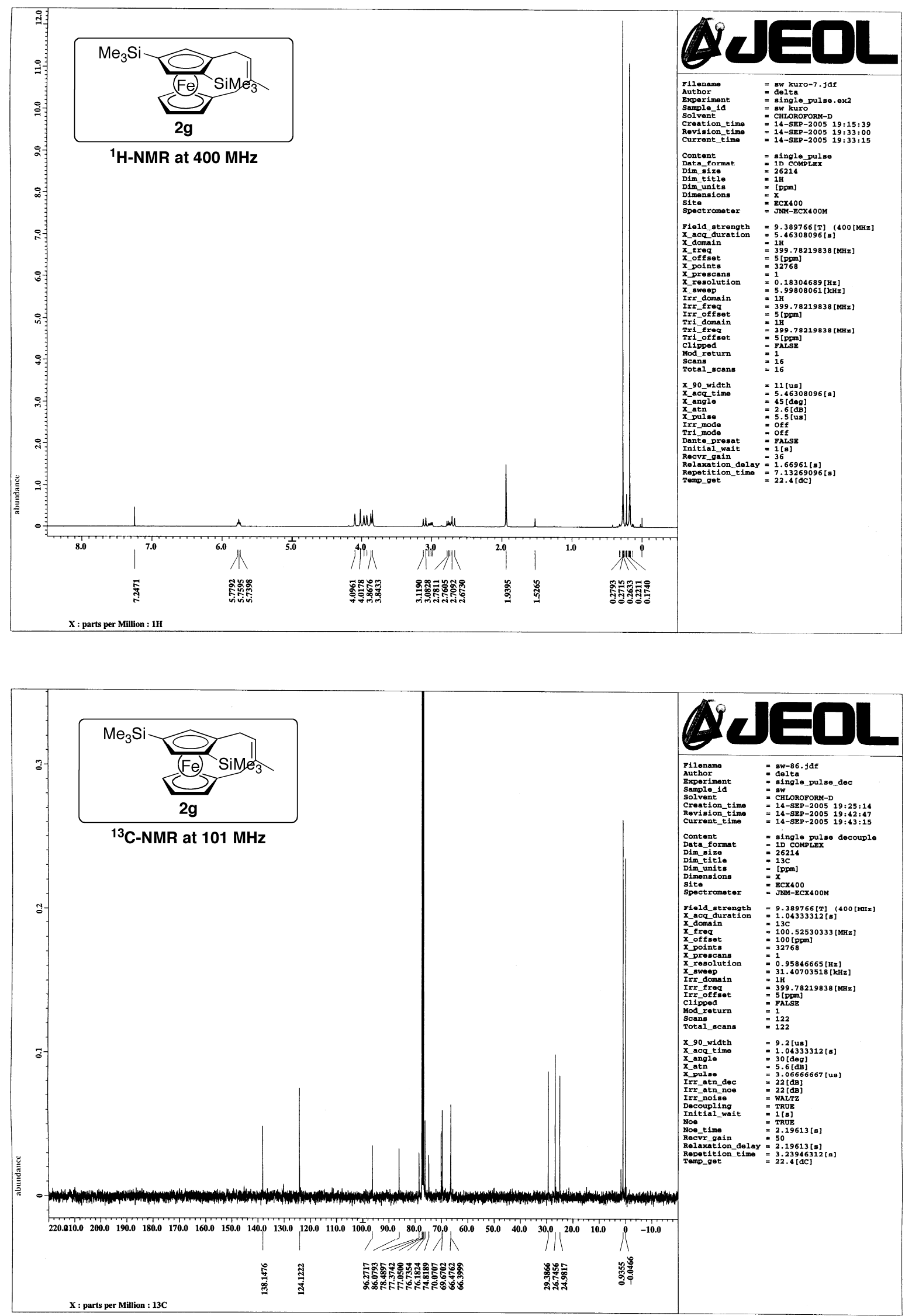

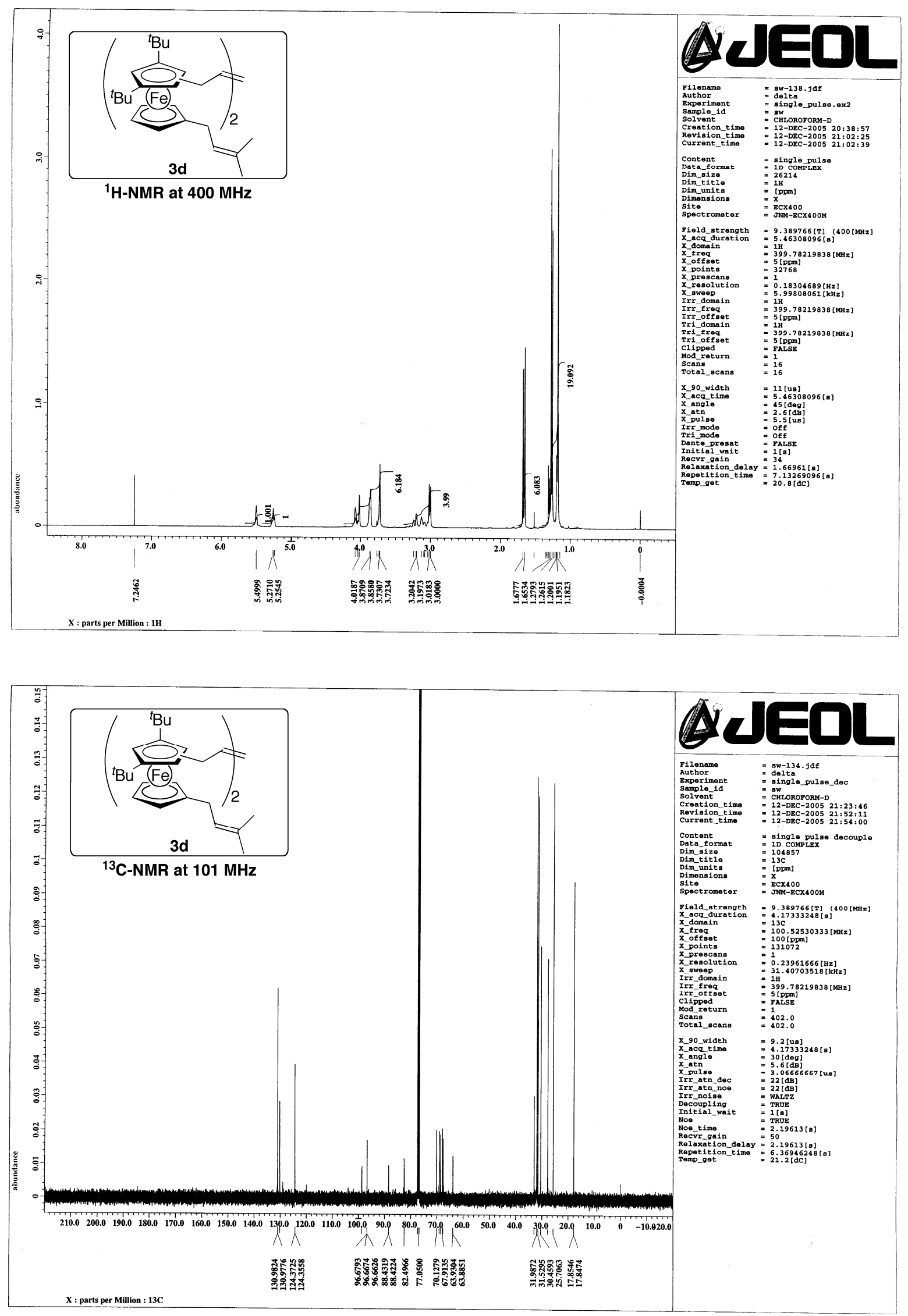